Background Systematic retinopathy (ROP) screening using dilated eye examination is currently performed in the neonatal intensive care unit (NICU). In France atropine $0.3 \%$ eye drops are currently used as a mydriatic agent, but no systematic assessments of clinical tolerance and efficacy have been described in the literature.

Purpose To assess the occurrence of clinical changes in infants at different time periods preceding and following atropine drops and eye examination, as well as the mydriatic efficacy of atropine in this context.

Materials and Methods Prospective pilot study, in one NICU (June-September 2012). Atropine $0.3 \%$ eye drops (one per eye) were instilled in accordance with French good practise guidelines. Data collection was performed at 3 consecutive periods (P1: $\mathrm{H}-24$ to $\mathrm{H} 0$ pre-atropine, $\mathrm{P} 2$ : $\mathrm{H} 0$ to $\mathrm{H}_{2} 4$ post-atropine, and $\mathrm{P} 3: \mathrm{H}_{2} 4$ to $\mathrm{H} 48$ postatropine), and included: abdominal distension, number of episodes of regurgitation or vomiting, necrotizing enterocolitis (NEC), somnolence, number of episodes of severe oxygen desaturation $(<70 \%)$, bradycardia ( $<100 \mathrm{bpm})$ and tachycardia $(>180 \mathrm{bpm})$. Assessment of efficacy was based on possibility for screening or not. McNemar's Exact Test and Wilcoxon-signed rank Test were used for the binary and continuous variables respectively. Significance was set at $\mathrm{p}<0.05$.

Results 18 children were screened (median gestational age at birth 27.2 weeks (IOR: 25.6-28.7), median corrected age 33.3 weeks (IOR: 32.3-34.3)). None of the variables showed a statistically significant difference between P1 and P3. Occurrence of abdominal distension $(\mathrm{P}=0.03)$, number of tachycardia $(\mathrm{P}=0.05)$ and oxygen desaturation events $(\mathrm{P}=0.03)$ were more frequent in $\mathrm{P} 2$ than in $\mathrm{P} 1$. No differences were found in thte occurrence of other variables between P1 and P2. No NEC was diagnosed. Effective pupillary dilatation was obtained in $78 \%$ of cases.

Conclusions Our study suggests that atropine is an efficient mydriatic agent for $\mathrm{ROP}$ screening dilated eye exam in preterm neonates. Type and timing of the symptoms in our study suggest systemic muscarinic effects of atropine. A reduction in the concentration of the atropine eye drops could improve tolerance.

No conflict of interest.

\section{CPC-011 AN AUDIT OF THE ADULT NUTRITION SUPPORT TEAM IN THE MANAGEMENT OF REFEEDING RISKS IN A UK TEACHING HOSPITAL}

doi:10.1136/ejhpharm-2013-000276.468

C Wong. Sheffield Teaching Hospitals NHS Foundation Trust, Pharmacy, Sheffield, UK

Background In June 2010 a report, 'A Mixed Bag - An enquiry into the care of hospital patients receiving parenteral nutrition', was published by the National Confidential Enquiry into Patient Outcome and Death (NCEPOD). They reviewed 870 adult case notes and found inadequate assessment and monitoring in $54 \%$ and metabolic complications in $40 \%$ of patients.

Purpose In early 2011 the adult nutrition support team (NST) wrote the clinical guidelines on the prevention and management of refeeding syndrome. The aim of this audit was to evaluate the impact of the NST in the management of refeeding risks in adult patients who required parenteral nutrition (PN).

Materials and Methods Adult PN records from April 2011 to March 2012 were assessed retrospectively by NST members using the NCEPOD Parenteral Nutrition Audit Tool. Microsoft Excel spreadsheets were used to record information on assessment and management of refeeding risks.

Results 259 PN records were reviewed. 54\% (140/259) patients were assessed and monitored by NST and 44\% (114/259) by critical care teams. The NST found a risk of refeeding syndrome in $31.4 \%$
(44/140) of patients prior to starting PN. The non-medical prescribers (NMPs) of the NST prescribed intravenous electrolyte infusions to $88.6 \%(39 / 44)$ of patients who were at high refeeding risk (see Table 1). Four patients had BMI less than $16 \mathrm{~kg} / \mathrm{m}^{2}$. The NMPs prescribed the lowest calorie feed $(1250 \mathrm{ml}$ Nutriflex Peri 5.7) and the infusion rate was reduced by $50 \%$ for the first two days in order to minimise metabolic complications.

Conclusions All adults referred to the NST for parenteral nutrition were reviewed and assessed for refeeding risk. The NMPs prescribed a range of intravenous electrolyte infusions to $88.6 \%$ of patients who were at high refeeding risk. This proactive prescribing approach by NMPs prevented the development of metabolic complications associated with low electrolyte levels prior to starting PN.

\section{Abstract CPC-011 Table 1}

\begin{tabular}{ll}
\hline Pre-PN electrolyte serum levels & Patient number (\%) \\
\hline Magnesium less than $0.7 \mathrm{mmol} / \mathrm{L}$ & $18(46.1 \%)$ \\
Phosphate less than $0.8 \mathrm{mmol} / \mathrm{L}$ & $11(28.2 \%)$ \\
Potassium less than $3.5 \mathrm{mmol} / \mathrm{L}$ & $4(10.3 \%)$ \\
Potassium and Magnesium below minimum levels & $6(15.4 \%)$ \\
\hline
\end{tabular}

No conflict of interest.

\section{CPC-012 AN ITALIAN COST-EFFECTIVENESS ANALYSIS OF PACLITAXEL ALBUMIN (NAB-PACLITAXEL) VS. CONVENTIONAL PACLITAXEL FOR METASTATIC BREAST CANCER PATIENTS: THE COSTANZA STUDY}

doi:10.1136/ejhpharm-2013-000276.469

${ }^{1} \mathrm{C}$ Lazzaro, ${ }^{2} \mathrm{R}$ Bordonaro, ${ }^{3} \mathrm{~F}$ Cognetti, ${ }^{3} \mathrm{~A}$ Fabi, ${ }^{4} \mathrm{~S}$ De Placido, ${ }^{4} \mathrm{G}$ Arpino, ${ }^{5} \mathrm{P}$ Marchetti, ${ }^{5} \mathrm{~A}$ Botticelli, ${ }^{6}$ P Pronzato, ${ }^{7}$ E Martelli. ' Studio di Economia Sanitaria, Milano, Italy; ${ }^{2}$ Public Hospital trust Garibaldi, Department of Medical Oncology, Catania, Italy; ${ }^{3}$ Regina Elena National Cancer Institute, Department of Medical Oncology A, Roma, Italy; ${ }^{4}$ Federico II University Hospital School of Medicine, Department of Endocrinology and Molecular and Clinical Oncology, Napoli, Italy; ${ }^{5}$ Sapienza University Hospital School of Medicine, Department of Medical Oncology, Roma, Italy; ${ }^{6}$ National Cancer Research Institute, Department of Medical Oncology A, Genova, Italy; '7Celgene Srl, Health Economics, Milano, Italy

Background Paclitaxel albumin (nab-paclitaxel) is a nanoparticle albumin-bound paclitaxel formulated with the aim of increasing the therapeutic index in metastatic breast cancer (MBC). When compared to conventional paclitaxel, nab-paclitaxel has reported longer time to progression, higher response and overall survival, lower incidence of neutropenia, no need for premedication and a shorter time of administration.

Purpose To investigate nab-paclitaxel's cost effectiveness vs. conventional paclitaxel for MBC patients in Italy.

Materials and Methods A Markov model with progression-free, progressed, and dead states was developed to estimate costs, outcomes and quality-adjusted life-years (OALYs) over 5 years from the Italian National Health Service (INHS) viewpoint. Patients were assumed to receive nab-paclitaxel $260 \mathrm{mg} / \mathrm{m}^{2} 3$-weekly (q3w) or conventional paclitaxel $175 \mathrm{mg} / \mathrm{m}^{2} \mathrm{q} 3 \mathrm{w}$. Data on health care resources consumption was collected from a survey performed on five Italian centres. Resources were valued at Euro $(€)$ 2011. Published utility weights were applied to health states to estimate the impact of response, disease progression and adverse events on OALYs. Three sensitivity analyses tested the robustness of the base case incremental cost-effectiveness ratio (ICER).

Results Compared to conventional paclitaxel, nab-paclitaxel gains an extra 0.165 OALYs (0.265 life-years saved) and incurs additional costs of $€ 2505$ per patient treated. This translates to an ICER of $€ 15,189$ (95\%CI: €11,891; €28,415). 